\title{
An idea of continuous thermographic monitoring of machinery
}

\author{
M. Fidali*
}

\author{
${ }^{*}$ Department of Fundamentals of Machinery Design - Silesian University of Technology, Gliwice, Poland
}

\section{Abstract}

Thermovision is more and more often used in machinery and apparatus diagnostics. There are different methods of evaluation of technical state on the basis of thermovision measurements. One of commonly applied are single or cyclic thermovision inspections. Another effective method is diagnosing of machinery during continuous monitoring of machines and devices. In both cases an thermogaphical image is a source of diagnostic information which can be extracted with the use of different methods of image analysis. In the article a way of machinery diagnosing on the basis of its continuous observation was presented. Verification of the proposed idea was carried out on the basis of data acquired during an active diagnostic experiment performed in the laboratory. Acquired images were analyzed and a huge set of obtained features was limited. In order to confirm whether the estimated features were optimal and the proposed idea could find application in machinery diagnostics classifications of known technical states were conducted.

\section{Introduction}

Thermographic measurements find broad applications in maintenance and assessment of technical states of machinery and apparatus, industrial processes, as well as manufacturing [7][11][14]. Thermographic inspections of technical objects are realized as a single or cyclic and consist in controlling of a current technical state of an object or identification of a pre-failure state and assessing a potential damage size. Technological progress and decreasing prices of industrial thermovision cameras have made the application of such apparatus possible to be applied to continuous monitoring and identifying states of machines and devices.

In case of continuous diagnostics of machinery a concept of a monitoring system based on thermovision images has been proposed (figure 1). The solution has allowed us to observe the object during its operation and estimate diagnostic parameters. The observation has being performed continuously. Because the parameters are functions of operation time they could allow us to detect early machine failures as well as draw prognoses related to a period of safe operation. Along this period all repair procedures could be prepared. The proposed system was characterized by a flexible structure and applied approaches, what has made it to be applied to different classes of machinery and apparatus.

A basis of a measurement part of the proposed monitoring system has been an infrared camera, which allowed observation of temperature distribution on a machine surface in range of an established field of view of the camera. The system consists also of a server, vision camera as well as sensors for observation of measurement path parameters. It was assumed that the system should also allow observation of additional signals by means of sensors (e.g. vibration) if such were installed in the observed object. The main part of the monitoring system has been the server where data were stored, analyzed and diagnostics decisions were sent to a machine operator. IR camera has delivered images with information about thermal state changes of the observed machine during its operation. The vision camera has been installed for observation of object illumination. Images from this camera were used for evaluation of influence of daylight on changes of thermogram features. Measurement path sensors (e.g. ambient temperature) delivered information about changes of surrounding condition which could also influence thermovision images. If the machine were equipped with additional sensors it would be useful to connect it to the system in order to correlate information and increase the system efficiency.

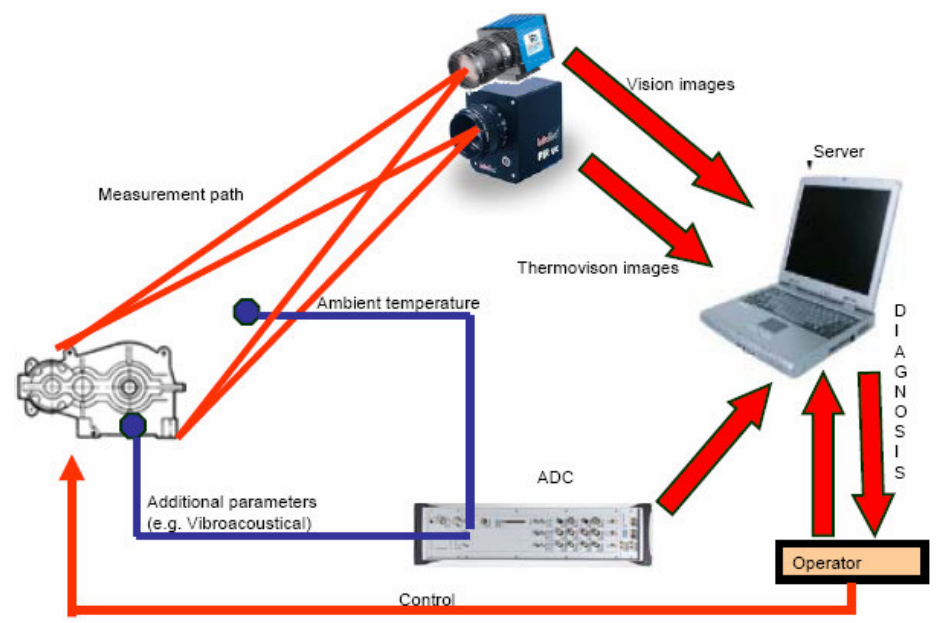

Fig. 1. Proposition of monitoring system for continuous thermographic monitoring 


\section{An idea of continuous thermal diagnostics of a machine}

Continuous thermovision diagnostics of objects is connected with conduction of necessary and systematic actions consisting in extracting of diagnostic data which is decoded in recorded thermographic images. In order to obtain relevant diagnostic information proper thermographic image processing methods should be applied. A thermographic image can be treated as a digital image described by a discrete function of temperature values of two variables $T(x, y)$, where $x$ and $y$ are coordinates of coordinate system determining spatial resolution of the image [7][11][15].

During continuous object observation with the use of a thermovision device, a sequence of thermographic images in time $t$ can be recorded. On the basis of acquired series of thermograms, a multidimensional thermographical signal ST(T $(x, y), t)$ is possible to be defined. If we consider a concept [3] of conventional real time partition into "micro" (dynamic) and "macro" (operation) time, often applied in machine diagnostics [2][8], then the thermographic signal can be defined in these both domains.

Taking into account "micro" and "macro" time concepts, the process of analysis of thermographic signals can be divided into two stages. The first stage is connected with thermogram analysis and feature extraction. It has enabled us to determine diagnostic signals in "micro" and/or "macro" time (figure 2).

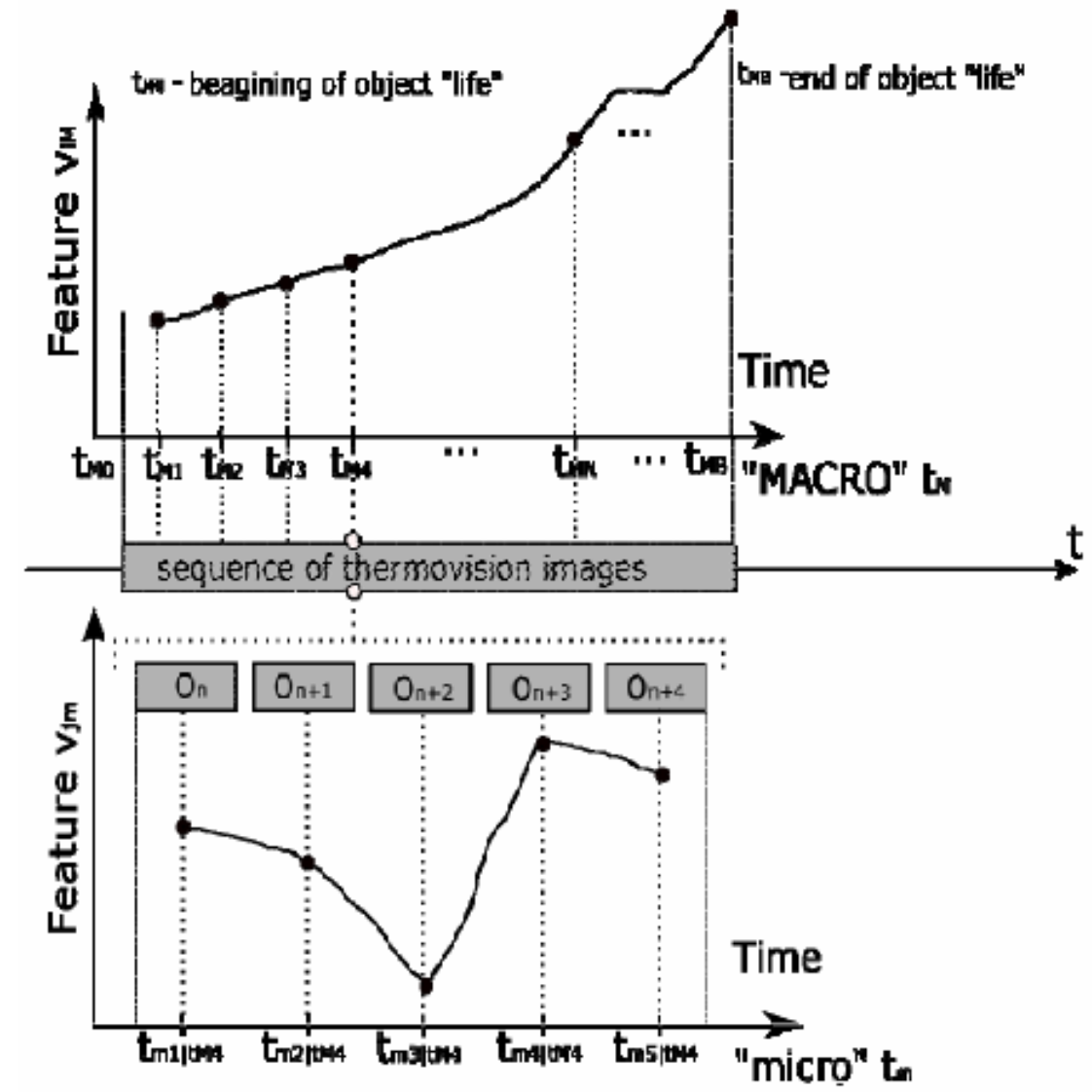

Fig. 2. Idea of analysis of thermographic signal in both "micro" and "macro" time domains

The second stage of analysis refers to the analysis of diagnostic signals which were determined at the first stage. For these purposes classical signal analysis methods could be applied.

\section{Analysis of thermovision images}

Image analysis is a wide domain of science which includes a lot of different kinds of methods. These methods are described in a variety of papers [6][7][11][17]. One of well known groups of image analysis methods have been methods dedicated to digital-image texture evaluation [7][10][11].

The major issue in texture analysis is feature extraction. This operation allows us to compute a characteristics of a digital image that let us describe numerically its texture properties. Feature extraction is the first stage of texture analysis. This operation can be used also for thermovision images and obtained results can be applied to such approaches as image discrimination, classification or object shape determination.

Among different methods of texture analysis statistical methods have being indicated as such approaches that make it possible to represent the texture indirectly by non-deterministic properties, which determine 
distributions and relationships between grey levels of an image [11]. Statistical methods allow us also to determine features of an image on the basis of different statistical characteristics calculated from [7][11]:

- histogram of an image

- gradient matrix

- run-length matrix

- co-occurrence matrix

- autoregression model.

\section{Active diagnostic experiment}

In order to verify usability of statistical methods for the analysis of thermovision images as well as for machinery diagnostics, an active diagnostic experiment has been carried out. The experiment has been performed with the use of a laboratory stand. The stand is located in the Laboratory of Technical Diagnostics of Department of Fundamentals of Machinery Design and consists of a laboratory model of rotating machinery and thermovision system (figure 3). The aim of the experiment was to acquire thermographic signals. As a result of diagnostic experiments series of thermograms recorded during object operation in different technical states have been obtained.

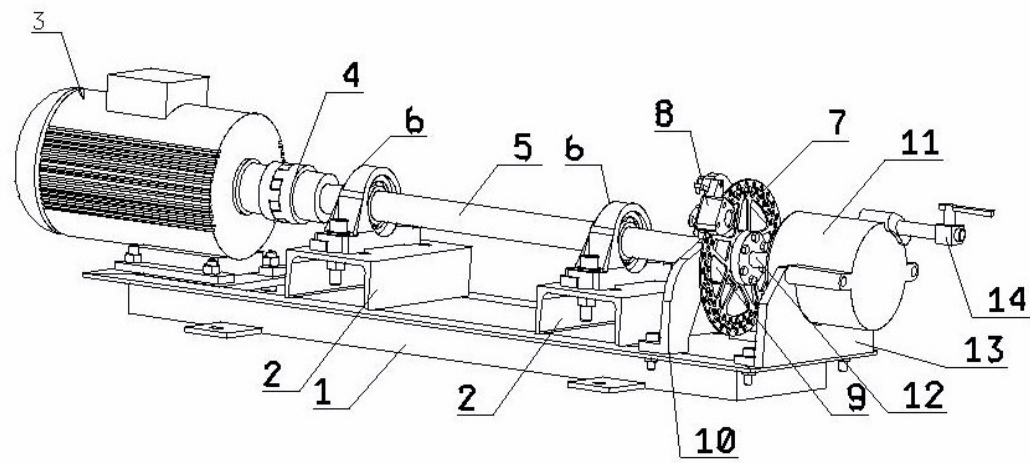
1-frame,
2-bearing support,
3-rotor,
4, 9, 12-couplings,
5 -shaft,
6-bearins set,
7-disk of brake,
8-brake clamp,
9-coupling,
10-brake support,
11- air pump,
13-pump support,
14- throttle valve

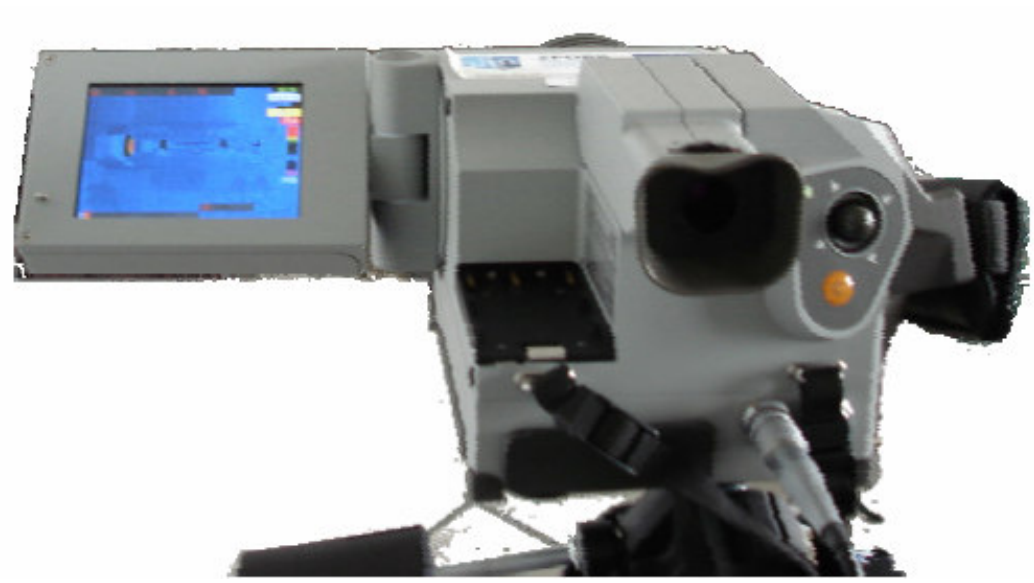

Infrared measurement system InfraTec VarioCam:

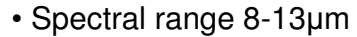

- Temperature range -402000oC

- Thermal resolution $100 \mathrm{mK}$

- Measurement accuracy +/- 2K

- Image resolution 320x240 px

Fig. 3. Investigated object and applied infrared camera

Thermographical images have been acquired during thermovision observations every 30 seconds period. The total number of recorded images has been 840 . The machine was working with rotation speed equal to 1150 rmp. The following technical states were simulated during machine operation:

$\mathrm{S} 1$ - machine without faults - 240 images

S2 $-50 \%$ throttling of air pump - 120 images

S3 $-90 \%$ throttling of air pump -120 images

S4 - $90 \%$ throttling of air pump + clearance of second bearing mounting -120 images

S5 - load of disk brake - 120 images

S6 - faulty bearing no $2-120$ images.

\section{Estimated features of thermographic images}

Recorded thermovision images have been analyzed with the use of selected statistical texture analyses. In this case specialized software MaZda dedicated to calculation of texture parameters (features) in digitized images has been applied. MaZda was originally developed in 1996 it the Institute of Electronics, Technical University of Lodz (TUL), Poland for mammogram analysis [15]. 
The software is able to analyze gray scale digital images with separated regions of interest and extract up to 259 different statistical parameters (features). Since that in case of thermogram analysis it was necessary to separate regions of interest (ROI) in the images. Five regions of interest have been defined (figure 4).

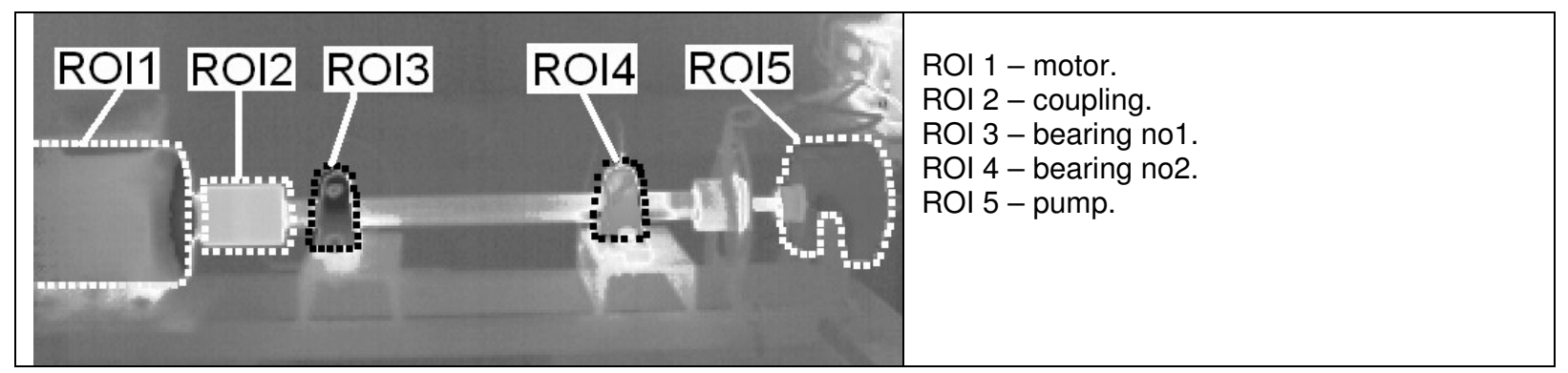

Fig. 4. Exemplary thermographic image with selected regions of interest (ROI)

For each region of interest (ROI) of every recorded image 259 features have been extracted. The following features [7][10] have been taken into consideration:

- 9 features estimated on the basis of histogram of the image (mean, variance, skewness, kurtosis and five histogram percentiles for $1 \%, 10 \%, 50 \%, 90 \%$, and $99 \%$ ),

- 5 features estimated on the basis of gradient matrix (absolute gradient mean, variance, skewness, kurtosis, and percentage of non-zero gradients),

- 220 features estimated on the basis of co-occurrence matrix . (11 features defined in calculated for matrices constructed for five distances between image pixels $(d=1,2,3,4$ and 5), and for the four directions as in the case of run-length matrix features),

- 20 features estimated on the basis of run length matrix (short run emphasis inverse moment, long run emphasis moment, gray level nonuniformity, run length nonuniformity and fraction of image in runs, separately for horizontal, vertical, $45^{\circ}$ and $135^{\circ}$ directions)

- 5 features estimated on the basis of autoregressive model parameters $(\theta \mathrm{i}, \mathrm{i}=1, \ldots, 4$ selected pixel-to-pixel relationship, noise standard deviation)

In figure 5 an exemplary plot of a diagnostic signal of mean temperature estimated for selected ROl's was presented. Values of selected feature were functions of numbers of consecutive recorded images. In the plot some technical states have been indicated (vertical lines). It can be observed that in some cases e.g. ROI 5 identification of changes of technical state was possible.

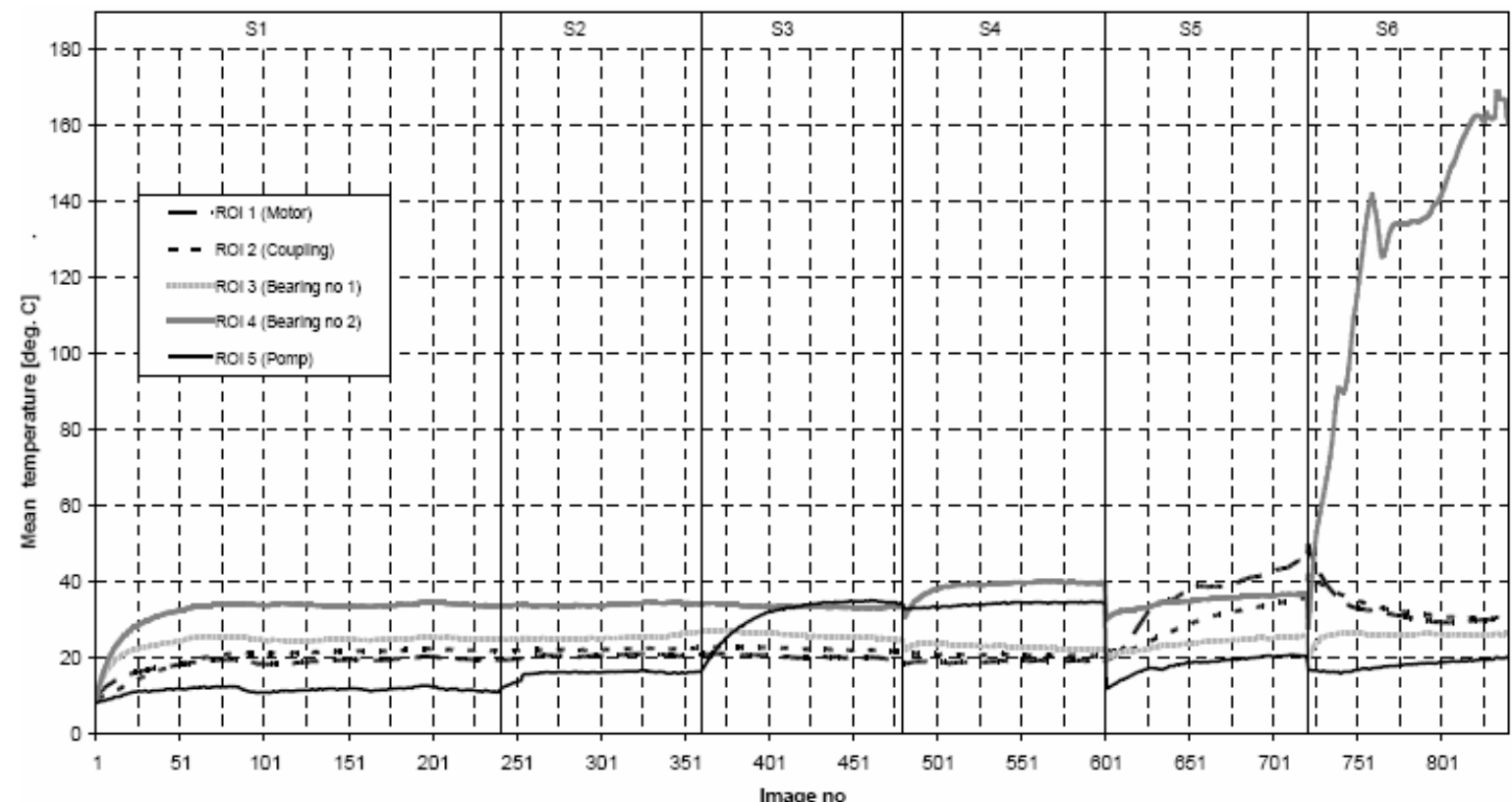

Fig. 5. Plot of diagnostic signal of mean value extracted from all considered region of interest as a function of numbers of consecutive recorded images. 


\section{Limitation of feature set}

In case of the proposed diagnostic method based on thermographic images it is important to find such relevant features which can be applied in effective manner for evaluation of machine technical state. This is not simple due to the large set of features which can be extracted from the image with the use of statistical methods of image analysis. On the basis of results of research reported in literature [4][8][14] it can be stated that an excessive number of input data entailed that information noise increased and efficiency of the classifier decreased. Therefore, it was important to identify a limited set of relevant and optimal diagnostic features.

There has been some ways of limitation of this set. One of an effective but time consuming method is comprehensive searching. Good results in optimization of feature space can be also obtained on the basis of discriminant analysis, and particularly extended Fisher's criterion [4][14]. Another way which gives very good results is the application of evolutionary algorithms [5].

In the article two methods implemented in the MaZda software were described. One method has been based on Fisher criterion [14] and another one, based on minimization of a classification error and correlation coefficient $(\mathrm{POE}+\mathrm{ACC})[15]$. It has been assumed that considered feature set was limited to 10 features. Results of feature space limitation were presented in tab. 1. One could observe that features on the basis of a cooccurrence matrix were dominating in the limited sets. There were also some features estimated on the basis of gradient matrix. Figures 6-9 show plots of selected features in functions of image numbers. One could observe that in most of considered ROls changes of parameters has been correlated to changes of machine technical state. Changes of technical state has been most visible for parameters estimated in the areas of motor and pump. Analysis of temperature in the area of bearing 3 allowed us to detect some changes of technical state. Less sensitive for technical changes was the coupling area where was hard to detect technical changes.

Table 1. Result of feature space limitation

\begin{tabular}{|l|l|}
\hline \multicolumn{1}{|c|}{ Fisher criterion } & \multicolumn{1}{c|}{ (POE+ACC) } \\
\hline$[\mathrm{S}(2,-2)$ Contrast]; & {$[\mathrm{S}(1,-1)$ Contrast]; } \\
{$[\mathrm{S}(3,3)$ Contrast]; } & {$[\mathrm{S}(0,2)$ Correlat]; } \\
{$[\mathrm{S}(3,-3)$ Contrast]; } & {$[\mathrm{S}(2,-2)$ Contrast] } \\
{$[\mathrm{S}(4,0)$ Contrast]; } & {$[\mathrm{S}(0,3)$ Contrast $]$} \\
{$[\mathrm{S}(0,4)$ InvDfMom]; } & {$[\mathrm{S}(0,4)$ Contrast]; } \\
{$[\mathrm{S}(4,4)$ Contrast]; } & {$[\mathrm{S}(0,4)$ SumOfSqs $]$} \\
{$[\mathrm{S}(5,0)$ Contrast]; } & {$[\mathrm{S}(4,4)$ Contrast $]$} \\
{$[\mathrm{S}(5,5)$ Contrast]; } & {$[\mathrm{S}(5,5)$ Contrast $]$} \\
{$[$ GrMean]; } & {$[45 d g r$ RLNonUni] } \\
[GrKurtosis]. & {$[$ GrKurtosis]. } \\
\hline
\end{tabular}

In comparison to plot of mean value shown in figure 5 selected features were informative. It can be also seen in plots of parameters computed in regions of bearings.

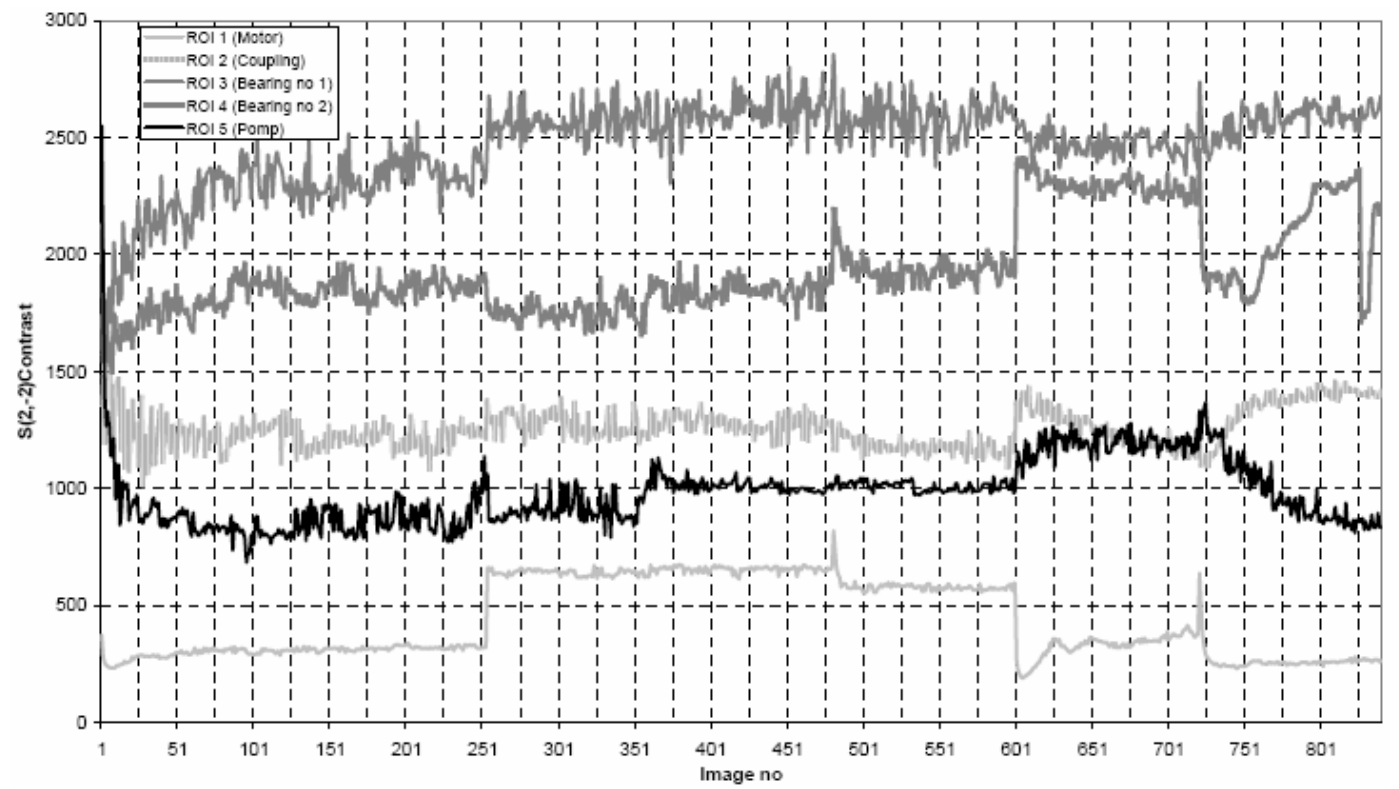

Fig. 6. Plot of diagnostic signal of Contrast $S(2,-2)$ value extracted from all considered region of interest as a function of numbers of consecutive recorded images. 


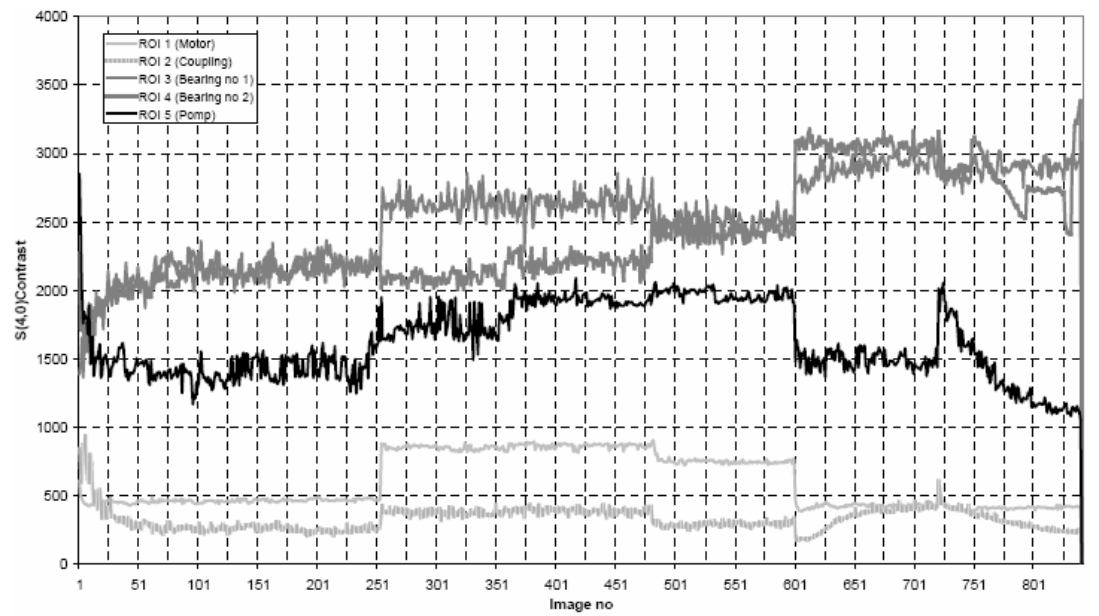

Fig. 7. Plot of diagnostic signal of Contrast $S(4,0)$ value extracted from all considered region of interest as a function of numbers of consecutive recorded images.

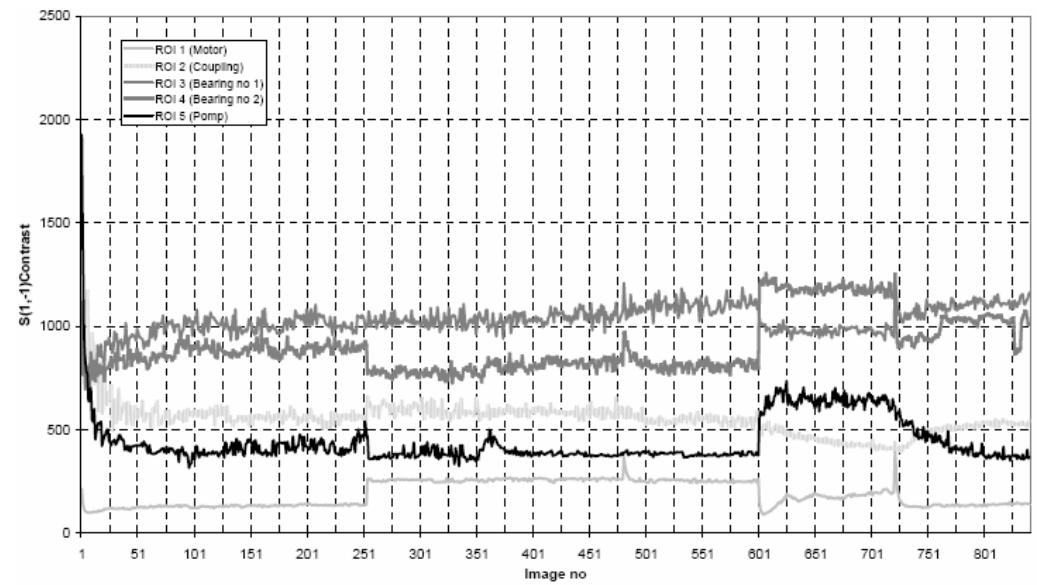

Fig. 8. Plot of diagnostic signal of Contrast $S(1,-1)$ value extracted from all considered region of interest as a function of numbers of consecutive recorded images.

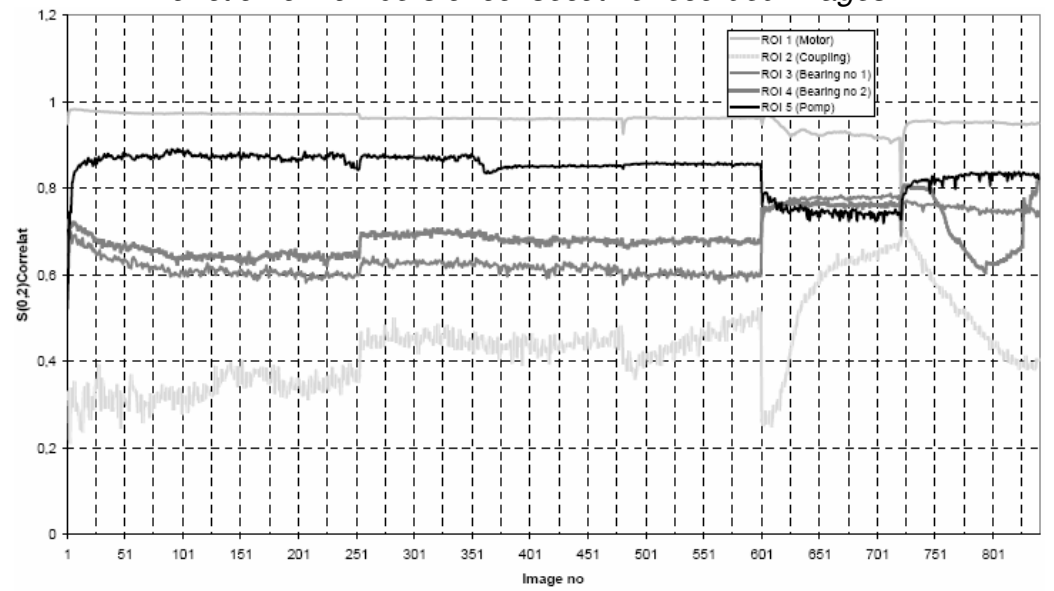

Fig. 9. Plot of diagnostic signal of Correlation $S(0,2)$ value extracted from all considered region of interest as a function of numbers of consecutive recorded images.

\section{Classification of machine technical state}

Visual evaluation of feature plots and classification of technical state on the basis of parameters plots is difficult and could be subjective. In case of the classification of machine technical state a neural network as a state classifier has been applied. The assumed neural network has been characterized by a simple structure due to small number of learning examples. On the basis of publications [1][8] it was assumed that total number of 
network parameters should be less than $1 / 5$ of the number of learning examples. A structure as well as parameters of the neural network have been shown in figure 10. The network included 4 layers: input (Lin), hidden (L1, L2) and output (Lout), and its parameters were shown in Table 2. All neurons in the input layer were characterized by weights values $=1$ and biases values $=0$. Parameters of neurons of the hidden and output layers were determined as a result of network training performed with the use of general purpose, scaled conjugate gradient backpropagation algorithm [12].

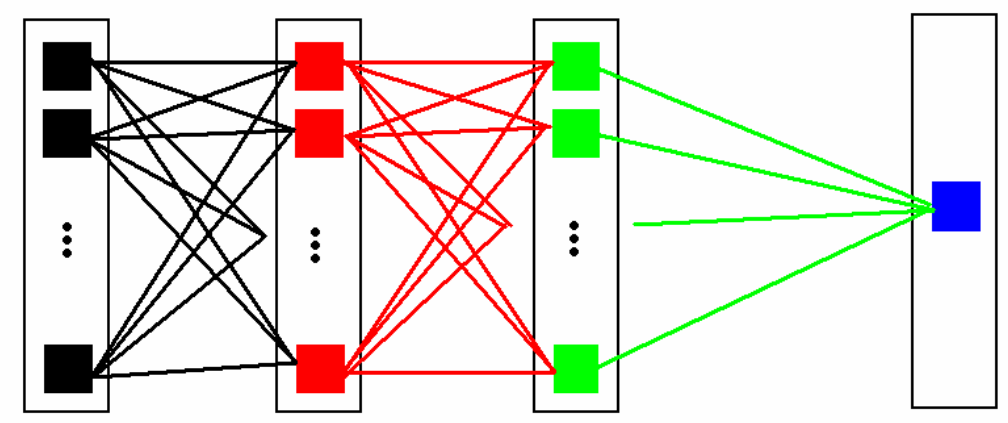

Fig. 10. The structure of neural network classifier

Table 2. Parameters of neural network

\begin{tabular}{|l|l|l|l|l|}
\hline layer & Lin & L1 & L2 & Lout \\
\hline number of neurons & 10 & 8 & 5 & 1 \\
\hline transfer function & linear & log-sigmoid & log-sigmoid & log-sigmoid \\
\hline
\end{tabular}

A number of learning examples was 588 which stands for $70 \%$ of the total number of examples (840). The efficiency of the classification was assessed by means of calculation of a relative number of correctly classified examples:

$$
\begin{aligned}
& \text { eff }=\frac{l_{1}}{l_{w}} \cdot 100 \% \\
& \text { where: } \quad l_{1}-\text { number of correctly classified examples, } \\
& \quad l_{w}-\text { number of all classified examples. }
\end{aligned}
$$

It was assumed that an acceptable error of the classification was $25 \%$. Each example, which exceeded this error was classified as incorrect. Results of the classification for two methods of feature selection were presented in table 3.

Table 3. Efficiencies of classifier

\section{Conclusions}

\begin{tabular}{|l|l|l|}
\hline \multirow{2}{*}{ ROI Id } & \multicolumn{2}{|c|}{ Classifier Efficiency [\%] } \\
\cline { 2 - 3 } & \multicolumn{2}{|c|}{ Feature selection method } \\
\cline { 2 - 3 } & Fisher criterion & (POE+ACC) \\
\hline 1 & 100,0 & 100,0 \\
\hline 2 & 95,63 & 83,73 \\
\hline 3 & 99,60 & 100,0 \\
\hline 4 & 96,82 & 98,01 \\
\hline 5 & 100,0 & 100,0 \\
\hline
\end{tabular}

In the article results of research which aim was to verify the proposed concept of evaluation of a technical state of an object on the basis of results of analysis of sequence of thermographical images were presented.

Thermograms recorded during an active diagnostic experiment have been analyzed with the use of different methods of statistical texture analysis. As the result of the analysis a set of 259 diagnostic features for each region of interest of each of 840 recorded images have been obtained. Five regions of interest have been considered. In order to search relevant features an Fisher criterion and minimization of a classification error and correlation coefficient $(\mathrm{POE}+\mathrm{ACC})$ has been applied. It has been assumed that the procedure of searching for the set of features was being performed until ten optimal features were found. The assessment of feature relevance has been performed on the basis of classification results and a simple neural network classifier has been applied.

The result of the research has shown that it was possible to find relevant features of thermographical images which can be used to identify technical state of a machine with very high efficiency. Obtained results have also shown that it was possible to classify the state of a technical object with a very high efficiency by means of Lin L1 L2 Lout statistical parameters of thermographical images. Results have revealed that in case of ROI no 2 (coupling) classifier efficiency was lower but also very good. The analysis of determined diagnostic signals has indicated possibilities of the application of statistical methods of thermogram analysis for determination of one 
dimensional diagnostic signal. The proposed concept can be used for identification of changes of technical states during machine operation.

Considering discussed results it can be stated that continuation of the research in this area is necessary. Further research should be focused on verification of other methods of image processing that could be useful for identification and classification of machine technical state. Selected methods of limitations of the set of estimated features do not affect significantly classification results. Statistical methods of thermogram analysis can be used for identification of changes of technical states during machine operation.

\section{REFERENCES}

[1] Aberdeen D., Baxter J., Edwards R.: 92\$/MFlops/s, Ultra-Large-Scale Neural-Network Training on a PIII Luster. citeseer.ist.psu.edu/aberdeen00cmflopss.html. (2000)

[2] Cempel Cz.: Fundamentals of vibroacoustical condition monitoring, in: A. Davies (ed), Handbook of Condition Monitoring. Techniques and Methodology, London, Chapman and Hall, 1998, pp. 324-353

[3] Cholewa W.: Method of machine diagnostics with application of fuzzy sets. Zeszyty Naukowe Nr 764. Politechnika Śląska, Gliwice 1983. (in polish)

[4] Ciupke K.: A comparative study on methods of reduction and selection of information in technical diagnostics. Mech. Syst. Signal Process. 2005 vol. 19, p. 919-938.

[5] Goldberg D.E.: Genetic Algorithms in Search, Optimization, and Machine Learning, Addison-Wesley Publishing Company, Inc., 1989.

[6] Gonzales C. R.: Wintz P.: Digital Image Processing. Addison-Wesley Publishing Company 1987.

[7] Haralick R., Shanmugam K., Dinstein I.: Textural Features for Image Classification, IEEE Trans. Systems Man Cybernetics, 3, 6 (1973) 610-621.

[8] Korbicz J., Kościelny J.M., Kowalczuk Z., Cholewa W. (Eds.): Fault diagnosis. Models, artificial inteligence, applications, Springer Verlag, Berlin Heidelberg, 2004

[9] Madura H.: Thermographic measurements in practice. Agencja wydawnicza PAK, Warszawa 2004. (In polish)

[10] Materka A., Strzelecki M., Lerski R., Schad L.: Evaluation of Texture Features of Test Objects for Magnetic Resonance Imaging, June 1999, Infotech Oulu Workshop on Texture Analysis in Machine Vision, Oulu, Finland, 13-19.

[11] Materka A., Strzelecki M.: Texture Analysis Methods - A Review. Technical University of Lodz, 1998, COST B11 Report.

[12] Matlab Help

[13] Minkina W.: Thermovision measurements. Devices and methods. Wydawnictwo Politechniki Częstochowskiej. Częstochowa 2004. (in polish)

[14] Sobczak W., Malina W.: Methods of information selection and reduction (in Polish). WNT, Warszawa 1985.

[15] Strzelecki M., Materka A.: European Project Cost B11 Quantitation Of Magnetic Resonance Image Texture. Zeszyty Naukowe 'Elektronika', vol. 4, 1999, 115-124 (in Polish).

[16] Thomas R.A., The thermography monitoring handbook. Coxmoor Publishing, Oxford, UK. 1999

[17] Wróbel Z., Koprowski R.: Thermographic image processing. Proceedings of VI krajowej konferencji Termografia i termometria w podczerwieni. Ustroń 2004. (in polish) 